OPEN ACCESS

Edited by:

Rachid Lahlali,

Ecole Nationale d'Agriculture

de Meknès, Morocco

Reviewed by:

Pinarosa Avato

University of Bari Aldo Moro, Italy

Fouad Mokrini,

National Institute for Agricultura

Research (Morocco), Morocco

*Correspondence:

Anupama Singh

anupamanil2000@gmail.com

Aditi Kundu

chem.aditi@gmail.com

Specialty section:

This article was submitted to

Plant Metabolism

and Chemodiversity,

a section of the journal

Frontiers in Plant Science

Received: 05 October 2020

Accepted: 20 November 2020

Published: 08 January 2021

Citation:

Kundu A, Dutta A, Mandal A, Negi L, Malik M, Puramchatwad $R$, Antil J, Singh A, Rao U, Saha S, Kumar R, Patanjali N, Manna S, Kumar A, Dash S and Singh PK (2021) A Comprehensive in vitro and in silico Analysis of Nematicidal

Action of Essential Oils.

Front. Plant Sci. 11:614143.

doi: $10.3389 /$ fpls.2020.614143

\section{A Comprehensive in vitro and in silico Analysis of Nematicidal Action of Essential Oils}

\author{
Aditi Kundu ${ }^{1 *}$, Anirban Dutta ${ }^{1}$, Abhishek Mandal ${ }^{1}$, Lalit Negi ${ }^{1}$, Monika Malik ${ }^{2}$, \\ Rajshekhar Puramchatwad ${ }^{2}$, Jyoti Antil'2, Anupama Singh ${ }^{1 *}$, Uma Rao $^{2}$, Supradip Saha', \\ Rajesh Kumar', Neeraj Patanjali', Suman Manna ${ }^{1}$, Anil Kumar ${ }^{3}$, Sukanta Dash ${ }^{3}$ and \\ P. K. Singh ${ }^{1}$
}

${ }^{1}$ Division of Agricultural Chemicals, ICAR-Indian Agricultural Research Institute, New Delhi, India, ${ }^{2}$ Division of Nematology, ICAR-Indian Agricultural Research Institute, New Delhi, India, ${ }^{3}$ Division of Design of Experiments, ICAR-Indian Agricultural Statistical Research Institute, New Delhi, India

Nematicidal potential of essential oils (EOs) has been widely reported. Terpenoids present in most of the essential oils have been reported responsible for their bioactivity though very less is known about their modes of action. In the present study, an in vitro screening of nine EOs, namely, Citrus sinensis (OEO), Myrtus communis (MTEO), Eucalyptus citriodora (CEO), Melaleuca alternifolia (TEO), Acorus calamus (AEO), Commiphora myrrha (MREO), Cymbopogon nardus (CNEO), Artemisia absinthium (WEO), and Pogostemon cablin (PEO) against Meloidogyne incognita revealed OEO, CNEO, and TEO as most effective with LC 50 39.37, 43.22, and $76.28 \mu \mathrm{g} \mathrm{ml}^{-1}$ respectively. EOs had varying compositions of mono- and sesquiterpenes determined by gas chromatography-mass spectrometry (GC-MS) analysis. The in silico molecular interactions screening of major EO constituents and the seven selected target proteins of the nematode indicated highest binding affinity of geraniol-ODR1 (odorant response gene 1) complex $\left(\Delta G=-36.9 \mathrm{kcal} \mathrm{mol}^{-1}\right)$, due to extensive $\mathrm{H}$-bonding, hydrophobic and $\pi$-alkyl interactions. The relative binding affinity followed the order: geraniolODR1 $>\beta$-terpineol-ODR1 $>$ citronellal-ODR1 $>$ l-limonene-ODR1 $>\gamma$-terpineneODR1. Taken together, the cumulative in vitro and computational bioefficacy analysis related to the chemoprofiles of EOs provides useful leads on harnessing the potential of EOs as bionematicides. The insight on biochemical ligand-target protein interactions described in the present work will be helpful in logical selection of biomolecules and essential oils for development of practically viable bionematicidal products.

Keywords: volatile oils, gas chromatography-mass spectrometry analysis, Meloidogyne incognita, molecular docking, odorant response gene 1

\section{INTRODUCTION}

Root knot nematodes, pose a major challenge to the global pest management programs due to devastating crop losses caused by these organisms (Sidhu et al., 2017). Among the root knot nematodes, Meloidogyne incognita is most abundant in tropical soils, barely sparing any crop family, and the most challenging part is to control its population below economic damage levels 
(Collange et al., 2011; de Freitas Silva et al., 2020). Synthetic recommended nematicides like carbofuran, fluopyram owing to their associated detrimental effects on the environment, non-target organisms besides phytotoxicity, necessitate safer approaches for nematode management in cropping systems (Westphal, 2011; Jones et al., 2017). To develop ecofriendly alternatives, a wide spectrum of plant metabolites with nematostatic and nematicidal actions has extensively been reported (Atolani and Fabiyi, 2020).

Phytochemicals have been extensively reported as potential sources of bioactive ingredients for the development of natural nematicides (Oka, 2001). Long-chain hydrocarbons, sulfur compounds, alkenes, furans, acetogenins, phenolics, saponins, etc., have been reported to be effective against various phytoparasitic nematodes (Aissani et al., 2018). Most of the phytochemicals have served as models for the identification of a lead molecule with potential commercial applications (Cantrell et al., 2012). Volatile organic compounds of botanical origin, most commonly found in essential oils, have particularly been recognized as highly effective against $M$. incognita (Aissani et al., 2015; Silva et al., 2018; Pedroso et al., 2019).

Plant essential oils (EOs) are complex mixtures of terpenoids and their oxygenated derivatives, produced by isoprenoid pathways (Tetali, 2019). Only $\sim 10 \%$ of the reported plant species produce EOs (Kalemba and Kunicka, 2003). Stored in secretory glands in epidermic cells, secretory hair, glandular trichomes, EOs play a key role in plant defense against biotic stresses (Bakkali et al., 2008). Known for their bioactive potential against diverse agriculturally important pests, EOs in numerous reports have been mentioned as very effective against $M$. incognita (Caboni et al., 2013). EOs of Acorus calamus and Pogostemon cablin have been tested for nematicidal activities (Perrett and Whitfield, 1995; Lee et al., 2009). EO from Eucalyptus citriodora was found highly toxic to M. incognita at $500 \mu \mathrm{l} \mathrm{ml}^{-1}$ by Pandey et al. (2000). Citrus sinensis EO was reported effective against the phytonematodes, M. incognita, Pratylenchus vulnus, and Xiphinema index (Avato et al., 2017). Similarly, EO of Myrtus communis was reported to kill $100 \%$ of $M$. incognita juveniles at $4,000 \mu \mathrm{ml}^{-1}$ concentration (Ardakani et al., 2013). Another study reported that EO of Melaleuca alternifolia was highly active against the larvae of Anisakis simplex at a concentration of $7 \mu \mathrm{mL}^{-1}$ (Andrés et al., 2012). The EO of Cymbopogon nardus tested against $M$. incognita exhibited moderate effectiveness in a study reported by Sinha et al. (2006). Similarly, the toxicity of Artemisia absinthium EO has been documented against $M$. incognita on the tomato plant (Amora et al., 2017). Promising nematicidal action of sesquiterpenes rich in the EO of Commiphora myrrha against juveniles of M. incognita was reported by Kong et al. (2006) and Ardakani et al. (2013).

A review of literature clearly showed that EO bioactivity evaluation against nematodes largely remained restricted so far to the evaluation of EOs against different plant parasitic nematodes. Emphasis on the correlation of their anti-nemic activity with chemical compositions and mechanism of interaction at molecular level with the possible target sites of action has remained lacking. Therefore, the present study was performed to characterize the chemical composition of the selected EOs, evaluate their bio-efficacy in vitro against $M$. incognita, and subject the most effective EOs to in silico analysis for a likely mode of action, using molecular docking and modeling approach.

\section{MATERIALS AND METHODS}

\section{Essential Oils}

Commercially available EOs (99\% purity) of different plants, namely, OEO (Citrus sinensis (L.) Osbeck; family Rutaceae, orange essential oil), MTEO (Myrtus communis L.; family Myrtaceae, myrtle essential oil), CEO (Eucalyptus citriodora L.; family Myrtaceae, citriodora essential oil), TEO (Melaleuca alternifolia L.; family Myrtaceae, tea tree oil), AEO (Acorus calamus L.; family Acoraceae, calamus essential oil), MREO [Commiphora myrrha (Nees) Engl; family Burseraceae, myrrh essential oil], CNEO (Cymbopogon nardus L. Rendle.; family Poaceae, citronella oil), WEO (Artemisia absinthium L.; family Asteraceae, wormwood essential oil), and PEO [Pogostemon cablin (Blanco) Benth.; family Lamiaceae, patchouli essential oil] were purchased from CDH Fine Chemicals (New Delhi, India) and Merck $^{\circledR}$ (New Delhi, India) and used without further purification.

\section{Chemicals and Reagents}

All the solvents used were of AR grade, purchased from Merck ${ }^{\circledR}$ (New Delhi, India). Surfactants, Atlas G5002 and Triton X100 were procured from Croda India Company Pvt. Ltd. (Navi Mumbai, India) and Loba Chemie Pvt., Ltd. (Mumbai, India), respectively. For GC-MS analysis, helium $(\mathrm{He})$ gas of high purity (99\%) was used.

\section{Gas Chromatography-Mass Spectrometry Analysis}

Volatile constituents of EOs were analyzed in GC-MS in a 7890A GC instrument (Agilent Technologies ${ }^{\circledR}$, United States) equipped with an HP-5MS column (30 m $\times 0.25 \mu \mathrm{m} ; / 0.25 \mu \mathrm{m}$, Agilent Co., United States) as stationary phase, which was directly connected to a triple axis HED-EM 5975C mass spectrometer (Agilent Co., United States). The injection volume was $1 \mu \mathrm{l}$ with flow mode in split control. Helium was used as carrier gas at a head pressure of $10 \mathrm{psi}$, and flow was set at $1 \mathrm{ml} \mathrm{min}^{-1}$. The GC-MS condition was programmed with the oven temperature initially held at $40^{\circ} \mathrm{C}$ for $1 \mathrm{~min}$, thereafter increased with a gradient of $3^{\circ} \mathrm{C} \mathrm{min}{ }^{-1}$, until the temperature reached to $120^{\circ} \mathrm{C}$ and held constant for $2 \mathrm{~min}$. The temperature was raised again with a gradient of $5^{\circ} \mathrm{C} \mathrm{min}^{-1}$ up to $220^{\circ} \mathrm{C}$ and held constant for $1 \mathrm{~min}$ and finally raised to $280^{\circ} \mathrm{C}$ with an increment of $4^{\circ} \mathrm{C} \mathrm{min}^{-1}$. The total run time of the analysis was $65 \mathrm{~min}$. The MS acquisition parameters were ion source temperature $180^{\circ} \mathrm{C}$, electron ionization $70 \mathrm{eV}$, full scan mode (50-550 AMU), transfer line temperature $280^{\circ} \mathrm{C}$, solvent delay $3 \mathrm{~min}$, and E.M voltage $1,380 \mathrm{~V}$. Compounds were identified by matching their mass spectra and fragmentation pattern using NIST (National Institute of Standards and Technologies) Mass Spectra Library. Further rentention indices (RI) have been calculated following Kovats (1978): 
TABLE 1 | Target sequences screened for in silico nematicidal activity.

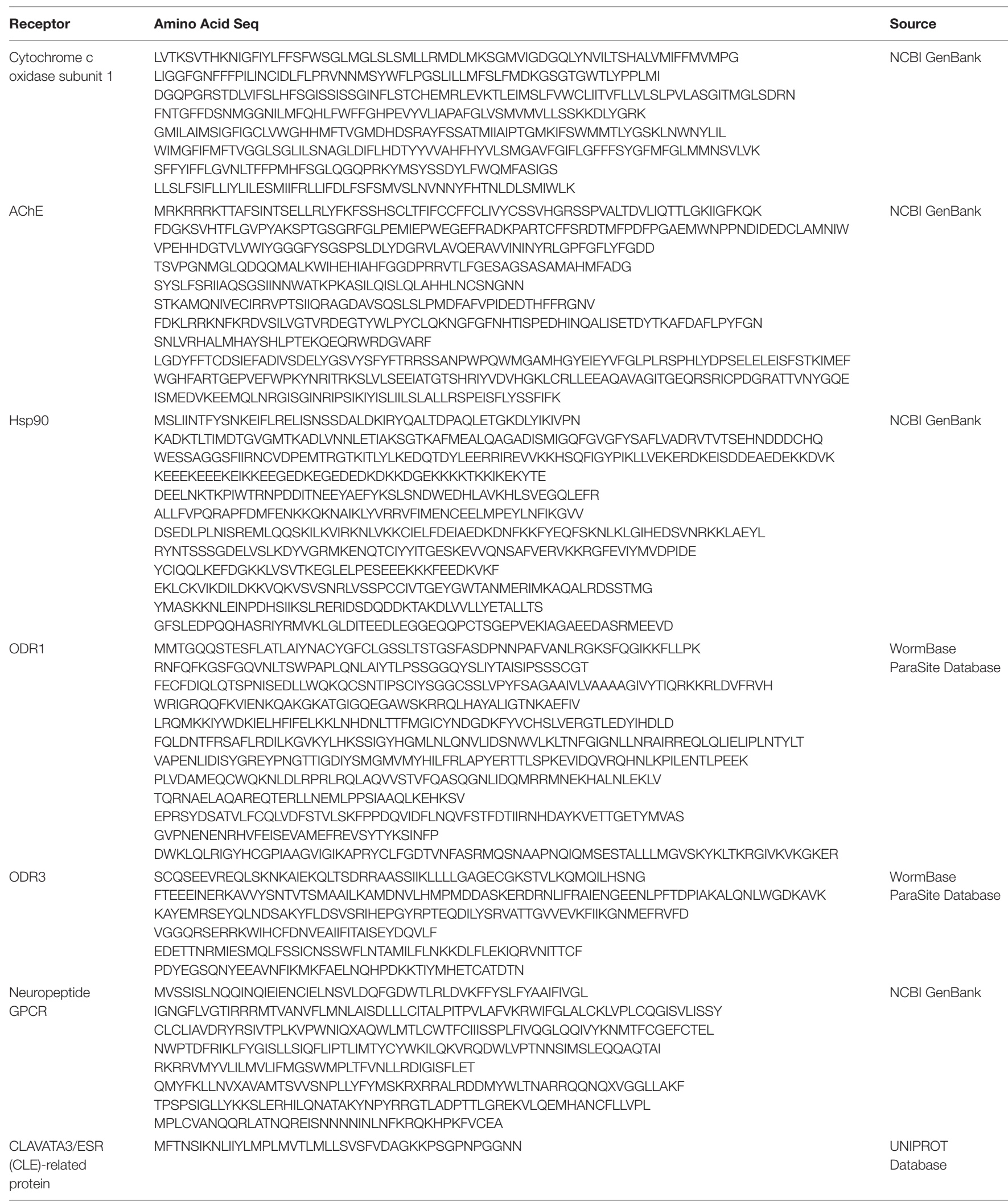

Meloidogyne incognita acetyl cholinesterase (AChE), M. incognita heat shock protein 90 (Hsp90), M. incognita odorant response gene-1 (ODR1), and M. incognita neuropeptide G-protein coupled receptor (nGPCR). 
TABLE 2 | Chemical composition of various EOs as analyzed in GC-MS and content (\%)c of constituents.

\begin{tabular}{|c|c|c|c|c|c|c|c|c|c|c|}
\hline Compounds $^{a}$ & $\mathbf{R} \mathbf{I}^{b}$ & OEO & MTEO & CEO & TEO & AEO & MREO & CNEO & WEO & PEO \\
\hline \multicolumn{11}{|l|}{$\begin{array}{l}\text { Monoterpene } \\
\text { hydrocarbons }\end{array}$} \\
\hline Thujene & 930 & nd & $0.2 \pm 0.1$ & nd & $2.3 \pm 0.1$ & nd & nd & nd & $0.1 \pm 0.0$ & nd \\
\hline o-Cymene & 937 & nd & nd & nd & nd & nd & nd & nd & $5.1 \pm 0.2$ & nd \\
\hline$\alpha$-Pinene & 939 & $1.6 \pm 0.1$ & $42.3 \pm 1.1$ & $0.1 \pm 0.0$ & $3.2 \pm 0.2$ & $0.2 \pm 0.1$ & $0.1 \pm 0.0$ & nd & $2.7 \pm 0.2$ & $0.2 \pm 0.0$ \\
\hline Camphene & 954 & nd & $4.2 \pm 1.0$ & $1.0 \pm 0.1$ & nd & $0.1 \pm 0.0$ & $0.3 \pm 0.0$ & nd & nd & nd \\
\hline$t$-Ocimene & 955 & $0.1 \pm 0.0$ & nd & nd & nd & nd & nd & nd & nd & nd \\
\hline Sabinene & 975 & $0.5 \pm 0.1$ & nd & nd & nd & nd & nd & nd & $5.6 \pm 0.3$ & nd \\
\hline$\beta$-Pinene & 979 & nd & $1.0 \pm 0.2$ & $0.2 \pm 0.0$ & $1.3 \pm 0.1$ & nd & $0.2 \pm 0.0$ & nd & $0.3 \pm 0.1$ & $0.3 \pm 0.0$ \\
\hline$\beta$-Myrcene & 991 & $2.2 \pm 0.2$ & nd & nd & $2.3 \pm 0.1$ & $0.1 \pm 0.0$ & nd & nd & $0.2 \pm 0.0$ & nd \\
\hline Phellandrene & 1,003 & nd & nd & nd & $0.6 \pm 0.0$ & nd & $0.1 \pm 0.0$ & nd & nd & nd \\
\hline p-Cymene & 1,013 & nd & nd & nd & $4.8 \pm 0.2$ & nd & nd & $0.1 \pm 0.0$ & $0.1 \pm 0.0$ & nd \\
\hline$\alpha$-Terpinene & 1,017 & nd & nd & nd & $8.7 \pm 0.6$ & nd & nd & nd & $0.9 \pm 0.3$ & $0.2 \pm 0.0$ \\
\hline I-Limonene & 1,029 & $93.2 \pm 2.3$ & nd & nd & nd & nd & nd & $5.7 \pm 0.5$ & nd & nd \\
\hline §-3-Carene & 1,033 & $0.4 \pm 0.1$ & $1.9 \pm 0.5$ & nd & nd & nd & nd & nd & nd & nd \\
\hline$\beta$-Ocimene & 1,051 & nd & nd & nd & nd & nd & $0.2 \pm 0.0$ & nd & $0.4 \pm 0.0$ & nd \\
\hline$\gamma$-Terpinene & 1,060 & nd & nd & nd & $17.5 \pm 1.1$ & nd & nd & nd & $1.1 \pm 0.2$ & nd \\
\hline$\alpha$-Terpinolene & 1,089 & nd & nd & nd & $2.8 \pm 0.3$ & nd & nd & nd & $1.1 \pm 0.2$ & nd \\
\hline \multicolumn{11}{|l|}{$\begin{array}{l}\text { Oxygenated } \\
\text { monoterpenes }\end{array}$} \\
\hline Fenchone & 1,008 & nd & nd & $2.3 \pm 0.3$ & nd & $0.1 \pm 0.0$ & nd & nd & nd & nd \\
\hline 1,8-Cineole & 1,035 & nd & $30.3 \pm 1.3$ & nd & $3.1 \pm 0.4$ & nd & nd & nd & $10.6 \pm 0.5$ & nd \\
\hline Limonene oxide & 1,087 & $0.2 \pm 0.0$ & nd & nd & nd & nd & nd & nd & nd & nd \\
\hline Linalool & 1,089 & $0.4 \pm 0.1$ & $7.6 \pm 0.9$ & nd & nd & $0.2 \pm 0.0$ & $1.7 \pm 0.3$ & $0.8 \pm 0.2$ & $6.4 \pm 0.4$ & nd \\
\hline Eucalyptol & 1,093 & nd & nd & $0.2 \pm 0.0$ & nd & nd & nd & nd & nd & nd \\
\hline$p$-Menth-8-en-2-ol & 1,090 & nd & nd & nd & $3.2 \pm 0.5$ & nd & nd & nd & nd & nd \\
\hline$t$-Thujone & 1,102 & nd & nd & nd & nd & nd & nd & nd & $0.3 \pm 0.0$ & nd \\
\hline Pulegol & 1,116 & nd & nd & nd & nd & nd & nd & $0.1 \pm 0.0$ & nd & nd \\
\hline Camphor & 1,146 & nd & nd & nd & nd & nd & nd & nd & $3.8 \pm 0.5$ & nd \\
\hline Citronellal & 1,148 & $0.1 \pm 0.0$ & nd & $81.9 \pm 1.1$ & nd & nd & nd & $31.5 \pm 1.1$ & nd & nd \\
\hline Isopulegol & 1,150 & nd & nd & nd & nd & nd & nd & $1.0 \pm 0.1$ & nd & nd \\
\hline$\beta$-Terpineol & 1,163 & nd & nd & nd & $35.7 \pm 1.2$ & nd & nd & nd & $16.2 \pm 0.9$ & nd \\
\hline Borneol & 1,169 & nd & nd & nd & nd & $0.1 \pm 0.0$ & nd & nd & $0.5 \pm 0.1$ & nd \\
\hline 4-Caranol & 1,185 & nd & nd & nd & nd & nd & nd & nd & $0.3 \pm 0.0$ & nd \\
\hline Decanal & 1,202 & $0.2 \pm 0.0$ & nd & nd & nd & nd & nd & nd & nd & nd \\
\hline Citronellol & 1,226 & nd & nd & $5.8 \pm 0.7$ & nd & nd & nd & $9.6 \pm 0.3$ & nd & nd \\
\hline Geraniol & 1,231 & nd & nd & nd & nd & nd & nd & $30.6 \pm 1.3$ & $0.6 \pm 0.1$ & nd \\
\hline Citral & 1,236 & nd & nd & $0.1 \pm 0.0$ & nd & nd & nd & nd & nd & nd \\
\hline Neral & 1,240 & nd & nd & nd & nd & nd & nd & $0.5 \pm$ & nd & nd \\
\hline Linalyl acetate & 1,257 & nd & $6.6 \pm 0.5$ & nd & nd & nd & nd & nd & nd & nd \\
\hline Geranial & 1,270 & nd & nd & nd & nd & nd & nd & $0.7 \pm$ & nd & nd \\
\hline Borneol acetate & 1,289 & nd & nd & nd & nd & nd & nd & nd & $26.6 \pm 1.7$ & nd \\
\hline Neryl acetate & 1,362 & nd & nd & nd & nd & nd & nd & $2.1 \pm 0.2$ & $0.7 \pm 0.1$ & nd \\
\hline Geranyl acetate & 1,383 & nd & nd & nd & nd & $0.1 \pm 0.0$ & nd & nd & $0.2 \pm 0.0$ & nd \\
\hline Thymol & 1,470 & nd & nd & nd & nd & nd & nd & nd & $0.3 \pm 0.0$ & nd \\
\hline \multicolumn{11}{|l|}{$\begin{array}{l}\text { Sesquiterpene } \\
\text { hydrocarbon }\end{array}$} \\
\hline$\delta$-Elemene & 1,343 & nd & nd & nd & nd & nd & $2.1 \pm 0.2$ & nd & $2.0 \pm 0.3$ & nd \\
\hline$\alpha$-Cubebene & 1,348 & nd & nd & nd & nd & $0.1 \pm 0.0$ & nd & nd & nd & nd \\
\hline$\alpha$-Copaene & 1,377 & nd & nd & nd & $0.1 \pm 0.0$ & nd & $0.2 \pm 0.0$ & nd & nd & nd \\
\hline$\beta$-Patchoulene & 1,382 & nd & nd & nd & nd & nd & nd & nd & nd & $5.8 \pm 0.5$ \\
\hline$\beta$-Elemene & 1,389 & nd & nd & nd & nd & nd & $4.8 \pm 0.7$ & $3.3 \pm 0.1$ & nd & $2.6 \pm 0.1$ \\
\hline$\alpha$-Gurjunene & 1,410 & nd & nd & nd & $2.1 \pm 0.1$ & nd & nd & nd & nd & $0.1 \pm 0.0$ \\
\hline
\end{tabular}


TABLE 2 | Continued

\begin{tabular}{|c|c|c|c|c|c|c|c|c|c|c|}
\hline Compounds $^{a}$ & $\mathrm{RI}^{b}$ & OEO & MTEO & CEO & TEO & AEO & MREO & CNEO & WEO & PEO \\
\hline$\beta$-Caryophyllene & 1,419 & nd & nd & $0.8 \pm 0.1$ & $0.3 \pm 0.0$ & $2.4 \pm 0.3$ & $0.5 \pm 0.1$ & nd & $8.2 \pm 1.0$ & $6.9 \pm 0.5$ \\
\hline$\alpha$-Guaiene & 1,430 & nd & nd & nd & nd & nd & nd & nd & $0.1 \pm 0.0$ & $40.6 \pm 1.3$ \\
\hline$\alpha$-Bergamotene & 1,436 & nd & nd & nd & nd & nd & $0.4 \pm 0.0$ & nd & nd & nd \\
\hline Aromadendrene & 1,443 & nd & nd & nd & $1.0 \pm 0.1$ & nd & nd & $1.4 \pm 0.1$ & nd & nd \\
\hline$\alpha$-Humulene & 1,455 & nd & $1.9 \pm 0.3$ & nd & nd & nd & $0.3 \pm 0.0$ & nd & nd & nd \\
\hline Farnesene & 1,457 & nd & nd & nd & nd & nd & nd & nd & $0.1 \pm 0.0$ & nd \\
\hline$\alpha$-Patchoulene & 1,460 & nd & nd & nd & nd & nd & nd & nd & nd & $10.7 \pm 0.5$ \\
\hline Neoisolongifolene & 1,462 & nd & nd & nd & nd & nd & nd & nd & nd & $1.0 \pm 0.1$ \\
\hline Alloaromadendrene & 1,466 & nd & nd & nd & $0.7 \pm 0.0$ & nd & nd & nd & nd & $4.4 \pm 0.2$ \\
\hline$\beta$-Salinene & 1,473 & nd & nd & nd & $0.3 \pm 0.0$ & nd & nd & nd & nd & nd \\
\hline$\gamma$-Muurolene & 1,477 & nd & nd & nd & $0.4 \pm 0.1$ & $1.2 \pm 0.2$ & nd & $0.3 \pm 0.0$ & nd & nd \\
\hline Germacrene D & 1,485 & nd & nd & nd & nd & nd & $1.2 \pm 0.1$ & $1.4 \pm 0.2$ & nd & nd \\
\hline Epi-bicyclophellandrene & 1,489 & nd & nd & nd & $0.4 \pm 0.1$ & nd & nd & nd & nd & nd \\
\hline Aciphyllene & 1,492 & nd & nd & nd & nd & nd & nd & nd & nd & $2.8 \pm 0.5$ \\
\hline$\alpha$-Bulnesene & 1,498 & nd & nd & nd & nd & nd & nd & nd & nd & $14.6 \pm 0.9$ \\
\hline$\delta$-Cadinene & 1,507 & nd & nd & nd & $1.6 \pm 0.2$ & nd & nd & nd & nd & nd \\
\hline Curcerene & 1,511 & nd & nd & nd & nd & nd & $23.9 \pm 2.0$ & nd & nd & nd \\
\hline$\alpha$-Panasinsene & 1,519 & nd & nd & nd & $0.2 \pm 0.0$ & nd & nd & nd & nd & nd \\
\hline Sesquiphellandrene & 1,523 & nd & nd & nd & $0.1 \pm 0.0$ & nd & nd & nd & nd & nd \\
\hline$\gamma$-Cadinene & 1,526 & nd & nd & nd & nd & nd & $0.3 \pm 0.0$ & $1.7 \pm 0.2$ & nd & nd \\
\hline$\alpha$-Bisabolene & 1,539 & nd & nd & $0.1 \pm 0.0$ & nd & nd & nd & nd & nd & nd \\
\hline$\alpha$-Calacorene & 1,547 & nd & nd & nd & nd & nd & $0.8 \pm 0.1$ & nd & nd & nd \\
\hline \multicolumn{11}{|l|}{$\begin{array}{l}\text { Oxygenated } \\
\text { sesquiterpene }\end{array}$} \\
\hline Methyl eugenol & 1,401 & nd & nd & nd & nd & $0.1 \pm 0.0$ & nd & $1.1 \pm 0.1$ & nd & nd \\
\hline Methyl isoeugenol & 1,455 & nd & nd & nd & nd & $3.1 \pm 0.2$ & nd & nd & nd & nd \\
\hline Elemol & 1,550 & nd & nd & nd & nd & nd & nd & $3.3 \pm 0.5$ & nd & nd \\
\hline Spathulenol & 1,561 & nd & nd & nd & nd & $1.7 \pm 0.1$ & nd & nd & nd & nd \\
\hline Caryophyllene oxide & 1,583 & nd & nd & $0.4 \pm 0.0$ & nd & $1.4 \pm 0.1$ & $0.4 \pm 0.0$ & nd & $0.2 \pm 0.0$ & $0.2 \pm 0.0$ \\
\hline Viridiforol & 1,588 & nd & nd & nd & $0.3 \pm 0.1$ & nd & $0.2 \pm 0.0$ & nd & nd & nd \\
\hline$\beta$-Asarone & 1,622 & nd & nd & nd & nd & $85.4 \pm 1.1$ & nd & nd & nd & nd \\
\hline Cadinol & 1,645 & nd & nd & nd & nd & nd & $0.9 \pm 0.1$ & $0.6 \pm 0.1$ & nd & nd \\
\hline$\gamma$-Eudesmol & 1,625 & nd & nd & nd & nd & nd & $0.2 \pm 0.1$ & nd & nd & nd \\
\hline$\beta$-Cudesmol & 1,649 & nd & nd & nd & $0.2 \pm 0.0$ & nd & $1.1 \pm 0.1$ & nd & nd & nd \\
\hline Patchoulol & 1,668 & nd & nd & nd & nd & nd & nd & nd & nd & $6.7 \pm 0.3$ \\
\hline Elemol acetate & 1,674 & nd & nd & nd & nd & nd & $1.2 \pm 0.2$ & nd & nd & nd \\
\hline$\alpha$-Bisabolol & 1,678 & nd & nd & nd & nd & nd & $0.2 \pm 0.0$ & nd & nd & nd \\
\hline$\alpha$-Asarone & 1,679 & nd & nd & nd & nd & $1.9 \pm 0.3$ & nd & nd & nd & nd \\
\hline Farnesol & 1,706 & nd & nd & $0.3 \pm 0.0$ & nd & nd & nd & nd & $3.0 \pm 0.2$ & nd \\
\hline Guaiol acetate & 1,721 & nd & nd & nd & $0.1 \pm 0.0$ & nd & nd & nd & nd & nd \\
\hline $\begin{array}{l}\text { Furanoeudesm-1,3- } \\
\text { diene }\end{array}$ & 2,091 & nd & nd & nd & nd & nd & $41.9 \pm 2.4$ & nd & nd & nd \\
\hline \multicolumn{11}{|l|}{ Others } \\
\hline $\begin{array}{l}\text { 2,6-Dimethyl-5- } \\
\text { heptenal }\end{array}$ & & nd & nd & $0.2 \pm 0.0$ & nd & nd & nd & nd & $\mathrm{Nd}$ & nd \\
\hline $\begin{array}{l}\text { 4,8-Dimethyl-3,7-non- } \\
\text { adienal }\end{array}$ & & nd & nd & $0.1 \pm 0.0$ & nd & nd & nd & nd & $\mathrm{Nd}$ & nd \\
\hline
\end{tabular}

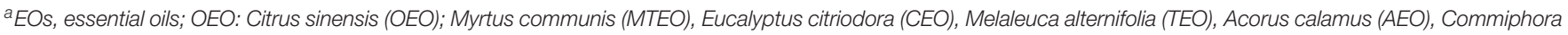

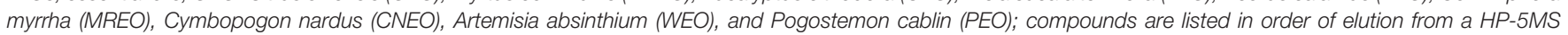

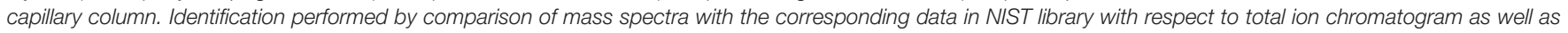
retention indices, calculated for alkanes $C_{9}$ to $C_{24}$ followed by comparison with the Adams (2007) report.

${ }^{b}$ Retention indices on the HP-5MS capillary column.

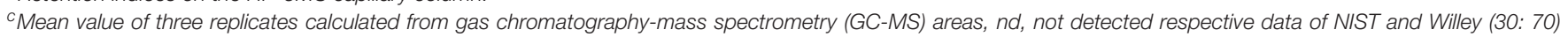

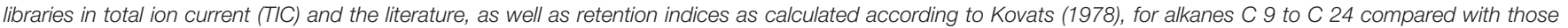
reported by Adams. 


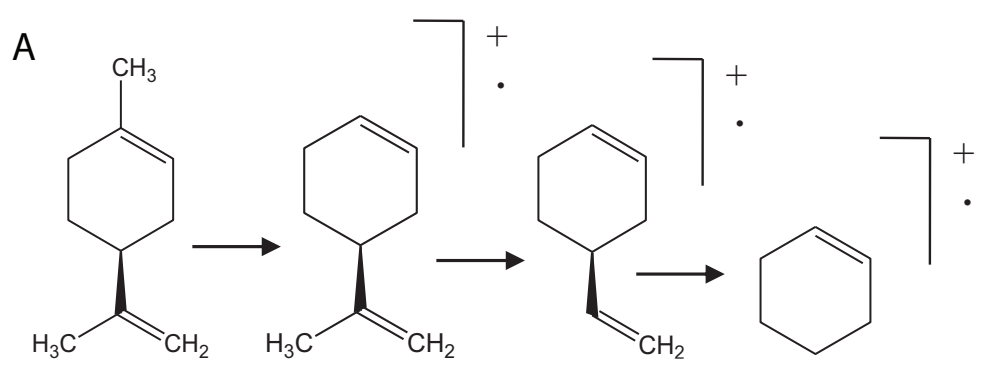
$\mathrm{m} / \mathrm{z} 136.2$
$\mathrm{m} / \mathrm{z} 122.2$
$\mathrm{m} / \mathrm{z} 108.2$
$\mathrm{m} / \mathrm{z} 71.2$<smiles>[B]CCC(C)CC(=O)CC(C)CCC=C(C)C</smiles>
$\mathrm{m} / \mathrm{z} 154.3$
$\mathrm{m} / \mathrm{z} 126.3$
$\mathrm{m} / \mathrm{z} 112.3$
$\mathrm{m} / \mathrm{z} 98.3$
$\mathrm{m} / \mathrm{z} 56.3$

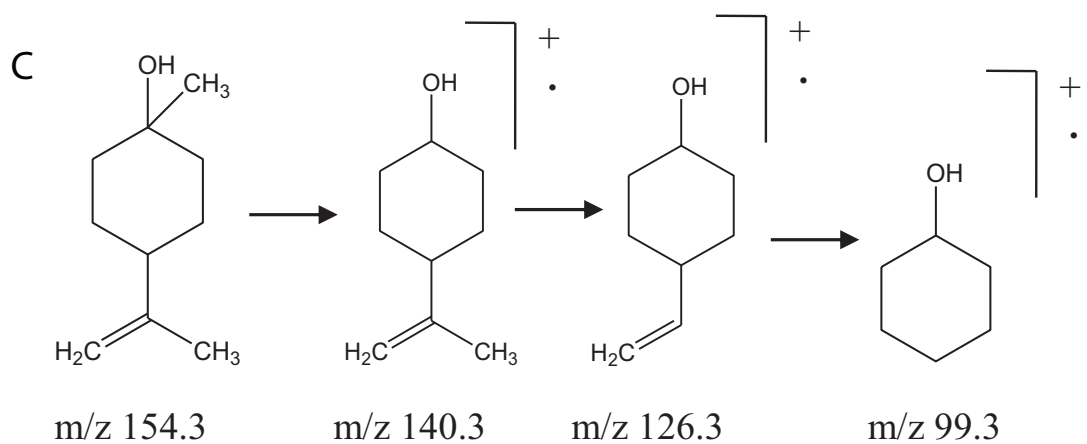

FIGURE 1 | Mass fragmentation pattern of (A) /-limonene, (B) citronellal, (C) and $\beta$-terpineol.

$\mathrm{RI}=100 * \mathrm{n}+\left[\log \left(\mathrm{RT}_{\text {compound }}-\mathrm{v}\right)-\log (\mathrm{RT}-\mathrm{v})\right] /\left[\log \left(\mathrm{RT}_{\text {larger }}\right.\right.$ alkane - $\left.\mathrm{v})-\log \left(\mathrm{RT}_{\text {smalleralkane }}-\mathrm{v}\right)\right]$

where $\mathrm{n}=$ the number of $\mathrm{C}$ in the smaller alkane, $\mathrm{RT}_{\text {compound }}=$ the retention time of the compound, $\mathrm{v}=$ the column void time, $\mathrm{RT}_{\text {larger alkane }}=$ the retention time of the larger alkane, and $\mathrm{RT}_{\text {smaller alkane }}=$ the retention time of the smaller alkane.

\section{Nematicidal Assay}

\section{Collection of Nematodes}

Nematode culture was maintained on infected tomato plants (var. Pusa Ruby) under greenhouse conditions. Second instar juveniles
$\left(\mathrm{J}_{2} \mathrm{~s}\right)$ of $M$. incognita were collected from roots of 21-day-old infected tomato seedlings. Nematode-infested soil was screened through water screening method following Cobb's sieving and decanting technique (Cobb, 1918). Further, nematode egg masses were picked up from the sterilized infected roots of tomato seedlings, transferred to fresh distilled water in Petri plates, and allowed to hatch under ambient condition of $27 \pm 1^{\circ} \mathrm{C}$ for 5 days. The hatched nematode juveniles travel through soft wet tissue placed on the wire of the Petri plate on the surface water (Julio et al., 2017). Nematode $\mathrm{J}_{2}$ s suspensions were combined and counted under light microscope. 
TABLE 3 | Chemical composition, number of compounds, group-wise classification of EO constituents.

\begin{tabular}{|c|c|c|c|c|c|c|c|c|c|}
\hline Classification & OEO & MTEO & CEO & TEO & AEO & MREO & CNEO & WEO & PEO \\
\hline Total identified composition (\%) & 98.9 & 96.0 & 93.5 & 93.3 & 98.2 & 83.2 & 95.8 & 97.7 & 97.1 \\
\hline Number of identified compounds & 10 & 9 & 14 & 26 & 16 & 24 & 19 & 29 & 15 \\
\hline Total monoterpene constituents (\%) & 98.9 & 94.1 & 91.6 & 85.5 & 0.9 & 2.6 & 82.7 & 84.1 & 0.7 \\
\hline Total sesquiterpene constituents (\%) & - & 1.9 & 1.6 & 7.8 & 97.3 & 80.6 & 13.1 & 13.6 & 96.4 \\
\hline Total hydrocarbons & 98.0 & 51.5 & 2.2 & 50.7 & 4.1 & 35.4 & 13.9 & 28.0 & 90.2 \\
\hline Total oxygenated compounds & 0.9 & 44.5 & 91.0 & 42.6 & 94.1 & 47.8 & 81.9 & 69.7 & 6.9 \\
\hline
\end{tabular}

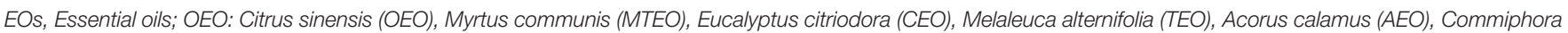
myrrha (MREO), Cymbopogon nardus (CNEO), Artemisia absinthium (WEO), and Pogostemon cablin (PEO).

\section{Preparation of Essential Oil Emulsions}

Primary stock emulsions $\left(10,000 \mu \mathrm{g} \mathrm{ml}^{-1}\right)$ of all EOs except PEO were prepared using Atlas G5002 surfactant $(2 \% \mathrm{w} / \mathrm{w})$. In the case of PEO, Triton X-100 (2\% w/w) was used. Each primary stock emulsion was diluted serially with surfactant solution to prepare secondary test emulsions of varying strengths $(1,000-$ $\left.10 \mu \mathrm{g} \mathrm{ml}^{-1}\right)$.

\section{Nematicidal Activity}

Nematicidal assay was conducted under in vitro condition to assess the activity of the EOs against $M$. incognita following a known method with slight modifications (Kundu et al., 2016). Treatments comprised of nine Eos, namely, OEO, MTEO, CEO, TEO, AEO, MREO, CNEO, WEO, and PEO. Aqueous suspension (1 $\mathrm{\mu l}$ ) containing $25 \mathrm{~J}_{2} \mathrm{~s}$ of $M$. incognita was added to each well of multiwell plates (15.6-mm diameter), each containing EO emulsion $(2 \mathrm{ml})$ of a particular test strength $(1,000-10 \mu \mathrm{g}$ $\mathrm{ml}^{-1}$ ). Surfactant solutions used to dissolve EOs were taken as corresponding negative controls. Each treatment was replicated thrice. Multiwell plates were incubated at $27 \pm 1^{\circ} \mathrm{C}$ and examined using a stereoscopic microscope at 24,48 , and 72 -h intervals. The numbers of dead vs alive juveniles in each treatment was recorded. Motionless nematodes with straight bodies were

TABLE $4 \mid$ LC $_{50}$ and $L C_{90}$ values $\left(\mu \mathrm{g} \mathrm{ml}^{-1}\right)$ of EOs against $M$. incognita, calculated for three exposure periods in test solutions.

\begin{tabular}{|c|c|c|c|c|c|c|}
\hline \multirow[t]{2}{*}{${ }^{*}$ EOs } & \multicolumn{3}{|c|}{${ }^{\star \star} \mathrm{LC}_{50}\left(\mu \mathrm{g} \mathrm{ml}^{-1}\right)$} & \multicolumn{3}{|c|}{${ }^{\star \star \star} \mathrm{LC}_{90}\left(\mu \mathrm{g} \mathrm{ml}^{-1}\right)$} \\
\hline & $24 \mathrm{~h}$ & $48 \mathrm{~h}$ & $72 \mathrm{~h}$ & $24 \mathrm{~h}$ & $48 \mathrm{~h}$ & $72 \mathrm{~h}$ \\
\hline OEO & 353.20 & 79.35 & 39.37 & 921.63 & 556.96 & 231.70 \\
\hline MTEO & $>1,000$ & 932.65 & 879.40 & $>1,000$ & $>1,000$ & $>1,000$ \\
\hline CEO & 746.48 & 330.41 & 124.50 & $>1,000$ & $>1,000$ & 987.42 \\
\hline TEO & 404.13 & 103.64 & 76.28 & $>1,000$ & 963.90 & 943.17 \\
\hline AEO & 524.45 & 90.11 & 85.23 & $>1,000$ & 353.21 & 310.92 \\
\hline MREO & $>1,000$ & $>1,000$ & $>1,000$ & $>1,000$ & $>1,000$ & $>1,000$ \\
\hline CNEO & 325.41 & 87.27 & 43.22 & 912.57 & 676.28 & 278.05 \\
\hline WEO & $>1,000$ & 937.52 & 734.72 & $>1,000$ & $>1,000$ & $>1,000$ \\
\hline PEO & $>1,000$ & 387.77 & 290.87 & - & $>1,000$ & $>1,000$ \\
\hline
\end{tabular}

${ }^{*}$ EOS, essential oils; OEO: Citrus sinensis (OEO), Myrtus communis (MTEO), Eucalyptus Citriodora (CEO), Melaleuca alternifolia (TEO), Acorus calamus (AEO), Commiphora myrrha (MREO), Cymbopogon nardus (CNEO), Artemisia absinthium (WEO), and Pogostemon cablin (PEO). ${ }^{* *} \mathrm{LC}_{50}\left(\mu \mathrm{g} \mathrm{mL} \mathrm{L}^{-1}\right)$ : Lethal concentration at $50 \%$ mortality of nematodes. ${ }^{* * *}{ }^{*} C_{90}\left(\mu \mathrm{mL} \mathrm{L}^{-1}\right)$ : Lethal concentration at $90 \%$ mortality of nematodes. counted. The revival test was done as described by Choi et al. (2007). Briefly, the motionless nematodes were teased with a needle followed by transfer to fresh wells containing deionized water. One drop of sodium hydroxide (1M) solution was added to check any movement. Mortality (\%) and corrected mortality (\%) of $\mathrm{J}_{2} \mathrm{~s}$ was calculated considering the mortality of juveniles in negative control.

\section{Molecular Docking and Simulation}

Based on the results of in vitro nematicidal assay and GC-MS analysis, major volatile constituents of OEO, CNEO, and TEO were selected out of nine EOs, for in silico ligand target protein interaction analysis.

\section{Selection of Protein}

Seven target proteins, namely, cytochrome $c$ oxidase subunit 1, AChE, Hsp90, ODR1, ODR3, neuropeptide GPCR, CLAVATA3/ESR (CLE)-related protein of $M$. incognita were selected as target receptors for the molecular docking studies. Cytochrome c oxidase subunit 1 is involved in the oxidative phosphorylation pathway, which is part of the energy metabolism. AChE regulates synaptic transmission and locomotion processes. The full functional activity of Hsp90 is gained in coordination with other co-chaperones, playing an important role in the folding of newly synthesized proteins, stabilization and refolding of denatured proteins during stress. ODR1 and ODR3 regulate chemosensory functions. Neuropeptide GPCR is associated in the regulation of movement of the parasite toward (or within) its host. CLAVATA3/ESR (CLE)-related protein plays an important role in the differentiation or division of feeding cells (syncytia) induced in plant roots during infection. (Ref. for each).

\section{Protein Preparation}

The hypothetical protein sequences were taken from the NCBI and UNIPROT database (Table 1). The BLAST servers ${ }^{1,2}$ were used to search and annotate the molecular and biological functions of the query sequences. The NCBI Blast tool and the PDB database were together used to identify the templates for modeling the secondary structures of the query sequences. Further homology modeling of the proteins was carried out using Modeller v 9.24.

\footnotetext{
${ }^{1}$ http://blast.ncbi.nlm.nih.gov

${ }^{2}$ https://parasite.wormbase.org//Tools/Blast
} 
TABLE 5 | In silico nematicidal activity of OEO, TEO, and CNEO oil constituents against $M$. incognita ODR1.

\begin{tabular}{|c|c|c|c|c|c|c|c|c|c|}
\hline NAME & Hbond & Hphob & Vwlnt & $\Delta G$ & Heavy_Atoms & $\log _{-} P$ & LE & Relative Percent (>10\%) & Oil Constituent \\
\hline Geraniol & -7.04 & -5.05 & -18.7 & -36.9 & 11 & 2.67 & 0.80 & 30.6 & CNEO \\
\hline Linalool & -5.08 & -5.43 & -21.8 & -36.2 & 11 & 2.67 & 0.79 & $<10.0$ & \\
\hline Geranial & -2.51 & -4.76 & -23.7 & -33.1 & 11 & 2.88 & 0.72 & $<10.0$ & \\
\hline$\beta$-Terpineol & -5.61 & -4.95 & -15.8 & -32.7 & 11 & 2.50 & 0.71 & 35.7 & TEO \\
\hline t-Ocimene & 0.00 & -5.89 & -20.9 & -28.9 & 10 & 3.48 & 0.69 & $<10.0$ & \\
\hline Neral & -3.61 & -4.76 & -21.0 & -31.0 & 11 & 2.88 & 0.67 & $<10.0$ & \\
\hline p-Menth-8-en-2-ol & -4.95 & -5.03 & -17.4 & -30.4 & 11 & 2.36 & 0.66 & $<10.0$ & \\
\hline Pulegol & -3.80 & -5.01 & -17.4 & -29.9 & 11 & 2.50 & 0.65 & $<10.0$ & \\
\hline 2,6-Dimethyl-5-heptenal & -2.58 & -4.64 & -18.5 & -26.8 & 10 & 2.57 & 0.64 & $<10.0$ & \\
\hline Citronellal & -2.66 & -4.79 & -20.5 & -29.3 & 11 & 2.96 & 0.64 & 31.5 & CNEO \\
\hline Limonene oxide & -1.86 & -4.30 & -15.6 & -28.7 & 11 & 2.52 & 0.62 & $<10.0$ & \\
\hline I-Limonene & 0.00 & -4.74 & -15.5 & -25.7 & 10 & 3.31 & 0.61 & 93.2 & OEO \\
\hline$\beta$-Myrcene & 0.00 & -5.42 & -21.5 & -24.6 & 10 & 3.48 & 0.59 & $<10.0$ & \\
\hline$\beta$-pinene & 0.00 & -4.49 & -14.3 & -24.2 & 10 & 3.00 & 0.58 & $<10.0$ & \\
\hline$\beta$-Pinene & 0.00 & -4.36 & -13.0 & -24.1 & 10 & 3.00 & 0.58 & $<10.0$ & \\
\hline$\beta$-Terpinolene & 0.00 & -5.20 & -18.5 & -24.0 & 10 & 3.45 & 0.57 & $<10.0$ & \\
\hline$\gamma$-Terpinene & 0.00 & -5.58 & -17.0 & -24.0 & 10 & 3.31 & 0.57 & 17.5 & TEO \\
\hline Para-cymene & 0.00 & -5.46 & -17.0 & -23.9 & 10 & 3.12 & 0.57 & $<10.0$ & \\
\hline 4,8-Dimethyl-3,7-non-adienal & -5.24 & -5.02 & -14.2 & -28.6 & 12 & 3.27 & 0.57 & $<10.0$ & \\
\hline Phellandrene & 0.00 & -5.43 & -16.4 & -23.6 & 10 & 3.16 & 0.56 & $<10.0$ & \\
\hline$\alpha$-Terpinene & 0.00 & -5.18 & -18.5 & -23.1 & 10 & 3.31 & 0.55 & $<10.0$ & \\
\hline Decanal & -2.45 & -5.13 & -19.8 & -25.4 & 11 & 3.33 & 0.55 & $<10.0$ & \\
\hline Isopulegol & -1.84 & -5.04 & -18.0 & -24.5 & 11 & 2.36 & 0.53 & $<10.0$ & \\
\hline Sabinene & 0.00 & -5.11 & -17.5 & -21.0 & 10 & 3.00 & 0.50 & $<10.0$ & \\
\hline$\delta$-Carene & 0.00 & -5.10 & -17.0 & -19.8 & 10 & 3.00 & 0.47 & $<10.0$ & \\
\hline Citronellol & -4.66 & -5.27 & -18.2 & -21.6 & 11 & 2.75 & 0.47 & 9.6 & CNEO \\
\hline Methyl eugenol & 0.00 & -5.64 & -23.9 & -25.4 & 13 & 2.43 & 0.47 & $<10.0$ & \\
\hline$\alpha$-Thujene & 0.00 & -5.13 & -16.3 & -19.2 & 10 & 3.00 & 0.46 & $<10.0$ & \\
\hline Neryl acetate & -1.56 & -5.92 & -21.4 & -26.7 & 14 & 3.24 & 0.45 & $<10.0$ & \\
\hline 1,8-Cineol & -1.16 & -4.88 & -14.3 & -20.1 & 11 & 2.74 & 0.44 & $<10.0$ & \\
\hline$\beta$-Caryophyllene & 0.00 & -5.72 & -17.1 & -26.3 & 15 & 4.73 & 0.42 & $<10.0$ & \\
\hline$\beta$-Eudesmol & -6.84 & -5.95 & -8.4 & -27.7 & 16 & 3.92 & 0.41 & $<10.0$ & \\
\hline$\gamma$-Cadinene & 0.00 & -5.60 & -19.5 & -25.3 & 15 & 4.58 & 0.40 & $<10.0$ & \\
\hline Epibicyclosesquiphellandrene & 0.00 & -5.66 & -17.8 & -25.2 & 15 & 4.58 & 0.40 & $<10.0$ & \\
\hline Sesquiphellandrene & 0.00 & -7.01 & -23.9 & -24.2 & 15 & 4.89 & 0.39 & $<10.0$ & \\
\hline Germacrene D & 0.00 & -5.71 & -16.6 & -23.9 & 15 & 4.89 & 0.38 & $<10.0$ & \\
\hline$\alpha$-Panasinsene & 0.00 & -5.45 & -15.3 & -23.4 & 15 & 4.56 & 0.37 & $<10.0$ & \\
\hline Cadinol & -5.20 & -6.03 & -10.5 & -24.8 & 16 & 3.78 & 0.37 & $<10.0$ & \\
\hline$\beta$-Selinene & 0.00 & -5.88 & -18.5 & -22.9 & 15 & 4.73 & 0.37 & $<10.0$ & \\
\hline Allo-aromadendrene & 0.00 & -5.50 & -14.2 & -21.2 & 15 & 4.27 & 0.34 & $<10.0$ & \\
\hline$\beta$-Elemene & 0.00 & -6.11 & -18.0 & -21.1 & 15 & 4.75 & 0.34 & $<10.0$ & \\
\hline$\gamma$-Muurolene & 0.00 & -5.99 & -18.2 & -20.5 & 15 & 4.58 & 0.33 & $<10.0$ & \\
\hline$\alpha$-Gurjunene & 0.00 & -5.43 & -15.1 & -20.3 & 15 & 4.42 & 0.32 & $<10.0$ & \\
\hline$\alpha$-Copaene & 0.00 & -5.91 & -16.7 & -19.2 & 15 & 4.27 & 0.31 & $<10.0$ & \\
\hline Viridiflorol & 0.00 & -5.54 & -13.7 & -20.1 & 16 & 3.47 & 0.30 & $<10.0$ & \\
\hline Guaiol acetate & -1.47 & -6.28 & -18.5 & -23.3 & 19 & 4.49 & 0.29 & $<10.0$ & \\
\hline Elemol & -3.74 & -6.28 & -12.7 & -19.7 & 16 & 3.94 & 0.29 & $<10.0$ & \\
\hline$\delta$-Cadinene & 0.00 & -6.78 & -10.1 & -16.3 & 15 & 4.73 & 0.26 & $<10.0$ & \\
\hline Aromadendrene & 0.00 & -5.79 & -11.9 & -15.3 & 15 & 4.27 & 0.24 & $<10.0$ & \\
\hline
\end{tabular}


TABLE 6 | Binding domains in the ODR1 target receptor.

\begin{tabular}{|c|c|c|}
\hline $\begin{array}{l}\text { Domain } \\
\text { Name }\end{array}$ & $\begin{array}{l}\text { Position } \\
\text { (Independent } \\
\text { E-value) }\end{array}$ & Description \\
\hline 1 & $549 \ldots \ldots \ldots$ & PF00211, Adenylate and \\
\hline Guanylate_cyc & $724(2.5 e-49)$ & $\begin{array}{l}\text { Guanylate cyclase catalytic } \\
\text { domain (Adenylate } \\
\text { cyclase-activating G } \\
\text { protein-coupled receptor } \\
\text { signaling pathway and cyclic } \\
\text { nucleotide biosynthetic } \\
\text { process) }\end{array}$ \\
\hline 2 & $261 \ldots \ldots \ldots$ & PF07714, Protein tyrosine and \\
\hline PK_Tyr_Ser_Thr & $479(1.2 \mathrm{e}-22)$ & $\begin{array}{l}\text { serine/threonine kinase (The } \\
\text { catalytic domain found in a } \\
\text { number of serine/threonine and } \\
\text { tyrosine-protein kinases is } \\
\text { represented by this entry) }\end{array}$ \\
\hline 3 & $498 \ldots \ldots \ldots$ & PF07701, Heme NO binding \\
\hline HNOBA & $541(0.0028)$ & $\begin{array}{l}\text { associated (This domain is } \\
\text { predicted to function in both } \\
\text { bacteria and animals as a } \\
\text { heme-dependent sensor for } \\
\text { gaseous ligands, and to } \\
\text { transduce various downstream } \\
\text { signals) }\end{array}$ \\
\hline
\end{tabular}

\section{Ligand and Receptor Preparation}

The molecular structures of the chemical constituents, referred hereafter as "ligands," of OEO, CNEO, and TEO were downloaded as.sdf file from PUBCHEM database ${ }^{3}$. The ligand structures were minimized using MM2 forcefield in Chem Draw Ultra 11.0 software [Cambridge Soft Corp., Cambridge, MA, United States (2009)] and used for molecular modeling studies. The ligand molecules were customized for docking using the Dock prep tool of Autodock Vina. Hydrogen molecules were added, and the incomplete side chains were replaced using Dunbrack rotamer library (Dunbrack, 2006). Charges were computed using ANTECHAMBER. AMBER ff14SB and Gasteiger charges were allotted to standard residues and to other residue types, respectively. Similarly, receptor molecules were prepared using the same tools except that the ANTECHAMBER was not employed. All the prepared ligand files were saved in the Mol2 format and the receptor files in the.pdb format.

\section{Molecular Docking Simulation}

The customized ligand and receptor molecules were used for docking in ICM Molsoft v. 2.8.ICM software, which performed adaptable ligand docking through global optimization of the energy function (Abagyan et al., 1994). The energy functions incorporated the internal energy of the ligand in view of the ECEPP/3 drive field, and van der Waals, hydrogen-holding, electrostatic and hydrophobic ligand/receptor association terms pre-ascertained on the lattice for computational proficiency (Bursulaya et al., 2003). Flexible ligand docking with the ICM software used Monte Carlo simulations to globally optimize a set of ligand internal coordinates in the space of grid

${ }^{3}$ pubchem.ncbi.nlm.nih.gov potential maps calculated for the protein pocket (Neves et al., 2012). Discovery Studio v. 4.1 Client was used to study the docked receptor-ligand interactions. The most favored docking conformation interactions of ODR 1 with geraniol, $\beta$-terpineol, citronellal, $l$-limonene, and $\gamma$-terpinene were analyzed on the basis of docking score, binding affinity, and interacting residues. The active site residues were identified, and depictions of all possible interactions in $3 \mathrm{D}$ and $2 \mathrm{D}$ poses were prepared using $\mathrm{DS}$ Visualiser v. 4.1.

In order to avoid affinity-based selection and optimization of larger ligands, the emphasis was given to compounds that most effectively utilized their atoms. In an attempt to measure the compound effectiveness, Hopkins et al. (2014) suggested an estimation of binding affinity of molecule, in terms of ligand efficiency (LE):

$$
\mathrm{LE}=\frac{[-2.303(\mathrm{RT}) \times \log K d]}{\mathrm{HA}}=\frac{-\Delta G}{\mathrm{HA}}
$$

where, $\Delta G$ is free-binding energy and HA is the number of ligand non-hydrogen atoms. LE is related to the amount and effectiveness of heavy atoms in a molecule toward complex formation. The average affinity contribution per atom was taken into consideration instead of considering the affinity of the whole compound. This enabled measuring the affinity of the corrected molecules with their size. In drug discovery modules, candidate molecules with $\mathrm{LE}$ values $\geq 0.3 \mathrm{kcal}$ per mole per heavy atom usually are taken ahead as lead molecule (Hopkins et al., 2014).

\section{Statistical Analysis}

The bioassay experiments were done in triplicate. The significance of the differences between variables was tested using one-way ANOVA. The means were compared using Duncan's multiple range test. Statistical significance was determined at $p<0.05$. Percent mortality data were subjected to probit analysis using Polo Plus software to determine lethal concentrations $\left(\mathrm{LC}_{50}\right.$ and $\mathrm{LC}_{90}$, expressed in $\mu \mathrm{g} \mathrm{ml}^{-1}$ ).

\section{RESULTS}

\section{Essential Oil Composition}

The compositions of EOs of OEO, MTEO, CEO, TEO, AEO, MREO, CNEO, WEO, and PEO were determined by comparing their mass spectra with data library, corresponding retention indices, and mass fragmentation patterns. The identified chemical constituents of the oils are listed in Table 2. The aromatic profile of most of the EOs showed dominance of one or two major constituents. Individually, $l$-limonene $(93.2 \pm 2.30 \%)$ was found to be most abundant in $\mathrm{OEO}$, along with $\beta$-myrcene $(2.2 \pm 0.2 \%)$, $\alpha$-pinene $(1.6 \pm 0.1 \%)$, sabinene $(0.5 \pm 0.1 \%)$, limonene oxide $(0.2 \pm 0.0 \%)$, and decanal $(0.2 \pm 0.0 \%)$. $l$-Limonene was confirmed based on its fragmentation pattern with characteristic daughter ion peaks of $\mathrm{m} / \mathrm{z} 136.2,108.2$, and 71.2 , generated due to sequential loss of methyl and ethyl moieties (Figure 1). Interestingly, OEO was found to contain only monoterpenes. The monoterpenic constituents of MTEO were identified as $\alpha$-pinene (42.3 $\pm 1.1 \%)$, 1,8-cineol (30.3 $\pm 1.3 \%)$, 
TABLE 7 | Molecular interaction details of flexible ligand docking of major oil constituents (the top 5) with the ODR1 receptor.

\begin{tabular}{|c|c|c|c|}
\hline Constituent & Bondbetween atoms & Distance & Type ofbonding \\
\hline \multirow[t]{6}{*}{ Geraniol } & :LYS599:HZ2 - Lig:Geraniol:O1 & 2.24581 & Conventional Hydrogen \\
\hline & :TYR607:HH - Lig:Geraniol:01 & 2.07546 & Conventional Hydrogen \\
\hline & Lig:Geraniol:H1 -:ASP589:OD1 & 2.07777 & Conventional Hydrogen \\
\hline & :TRP220 - Lig:Geraniol:C9 & 4.53747 & Pi-Alkyl Hydrophobic \\
\hline & :PHE585 - Lig:Geraniol:C10 & 4.62898 & Pi-Alkyl Hydrophobic \\
\hline & :TYR607 - Lig:Geraniol:C8 & 5.20422 & Pi-Alkyl Hydrophobic \\
\hline \multirow[t]{4}{*}{$\beta$-Terpineol } & Lig:ß-Terpineol:H1 -:ASN340:OD1 & 2.14644 & Conventional Hydrogen \\
\hline & 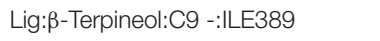 & 5.19655 & Alkyl Hydrophobic \\
\hline & :TRP220 - Lig: $\beta$-Terpineol & 5.04265 & Pi-Alkyl Hydrobhobic \\
\hline & :TRP220 - Lig:ß-Terpineol:C9 & 4.59553 & Pi-Alkyl Hydrobhobic \\
\hline \multirow[t]{7}{*}{ Citronellal } & :ASN582:HD22 - Lig:Citronellal:O1 & 1.86408 & Conventional Hydrogen \\
\hline & :ASN582:HA - Lig:Citronellal:O1 & 2.5931 & Carbon Hydrogen \\
\hline & Lig:Citronellal:C8 -:LEU338 & 5.31917 & Alkyl Hydrophobic \\
\hline & Lig:Citronellal:C8 -:ILE389 & 5.35293 & Alkyl Hydrophobic \\
\hline & Lig:Citronellal:C9 -:ILE389 & 5.26424 & Alkyl Hydrophobic \\
\hline & :TRP220 - Lig:Citronellal:C9 & 5.02254 & Pi-Alkyl Hydrobhobic \\
\hline & :PHE585 - Lig:Citronellal:C10 & 4.31251 & Pi-Alkyl Hydrobhobic \\
\hline \multirow[t]{7}{*}{ I-Limonene } & :LEU349 - Lig: I-Limonene & 5.48522 & Alkyl Hydrophobic \\
\hline & :PRO385 - Lig: /-Limonene & 4.65347 & Alkyl Hydrophobic \\
\hline & Lig: I-Limonene:C10 -:VAL383 & 4.26576 & Alkyl Hydrophobic \\
\hline & Lig: I-Limonene:C10 -:PRO385 & 3.65949 & Alkyl Hydrophobic \\
\hline & Lig: /-Limonene:C4 -:LEU349 & 4.67353 & Alkyl Hydrophobic \\
\hline & Lig: I-Limonene:C4 -:VAL383 & 4.5661 & Alkyl Hydrophobic \\
\hline & :TRP347 - Lig:Limonene & 5.44615 & Pi-Alkyl Hydrobhobic \\
\hline \multirow[t]{11}{*}{$\gamma$-Terpinene } & :PRO534 - Lig: $\gamma$-Terpinene & 4.26992 & Alkyl Hydrophobic \\
\hline & :PRO535 - Lig: $\gamma$-Terpinene & 5.10312 & Alkyl Hydrophobic \\
\hline & :ALA597 - Lig: $\gamma$-Terpinene & 3.68262 & Alkyl Hydrophobic \\
\hline & :ALA597 - Lig: $\gamma$-Terpinene:C8 & 4.33181 & Alkyl Hydrophobic \\
\hline & :LYS599 - Lig: $\gamma$-Terpinene & 5.25154 & Alkyl Hydrophobic \\
\hline & :ALA610 - Lig: $\gamma$-Terpinene:C9 & 4.07633 & Alkyl Hydrophobic \\
\hline & 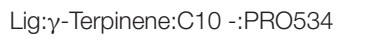 & 4.0165 & Alkyl Hydrophobic \\
\hline & Lig: $\gamma$-Terpinene:C10 -:VAL613 & 4.30711 & Alkyl Hydrophobic \\
\hline & Lig: $\gamma$-Terpinene:C10 -:PR0614 & 4.12284 & Alkyl Hydrophobic \\
\hline & Lig: $\gamma$-Terpinene:C8 -:PRO535 & 4.64633 & Alkyl Hydrophobic \\
\hline & :TYR672 - Lig: $\gamma$-Terpinene:C10 & 5.24394 & Pi-Alkyl Hydrobhobic \\
\hline
\end{tabular}

linalool (7.6 $\pm 0.9 \%)$, and linalyl acetate $(6.6 \pm 0.5 \%)$. GCMS analysis of TEO showed several peaks corresponding to 27 mono and sesquiterpenoids, comprising $93.3 \%$ of the total oil. Monoterpenes (43.5\%) and their oxygenated derivatives (42.6\%) were found to be the most abundant. Among monoterpenes, $\beta$ terpineol $(35.7 \pm 1.2 \%)$ was identified as the major constituent followed by $\gamma$-terpinene $(17.5 \pm 1.1 \%)$, $\alpha$-terpinene $(8.7 \pm 0.6 \%)$, p-cymene $(4.8 \pm 0.2 \%), \alpha$-pinene $(3.2 \pm 0.2 \%)$, p-menth-8-en-2$\mathrm{ol}(3.2 \pm 0.5 \%)$, and 1,8 -cineol $(3.1 \pm 0.4 \%)$. Besides, $\alpha$-gurjunene $(2.1 \pm 0.1 \%)$ and $\delta$-cadinene $(1.6 \pm 0.2 \%)$ were identified as the major sesquiterpenes.

Analysis of volatiles of CEO and CNEO revealed the presence of various terpenes, representing $93.5 \%$ and $95.8 \%$ of the total oil composition, respectively. These oils were characterized by the presence of predominant acyclic monoterpene aldehyde and citronellal with its respective contents of $81.9 \pm 1.1 \%$ and $31.5 \pm 1,1 \%$, in two oils. Both CEO and CNEO showed higher content of oxygenated compounds, in which the former attributed an appreciably higher content primarily of $91.3 \%$ oxygenated monoterpenes. Similarly, CNEO mainly contained oxygenated terpenoids (81.9\%) and hydrocarbons (13.9\%). Except citronellal, other constituents of CEO were citronellol $(5.8 \pm 0.7 \%)$ and fenchone $(2.3 \pm 0.3 \%)$, while CNEO contained geraniol (30.6 $\pm 1.3 \%)$, citronellol (9.6 $\pm 0.3 \%)$, $l$-limonene $(5.7 \pm 0.5 \%), \beta$-elemene $(3.3 \pm 0.1 \%)$, and neryl acetate $(2.1 \pm 0.2 \%)$.

GC-MS analysis of AEO showed identification of 16 mono and sesquiterpenes, accounting for $98.2 \%$ of the total oil. Oxygenated terpenes were the major constituents (94.1\%) of the oil with the $\beta$-asarone being the highest contributor $(85.4 \pm 1.1 \%)$. Methyl isoeugenol $(3.1 \pm 0.2 \%)$, caryophyllene $(2.4 \pm 0.3 \%), \alpha$-asarone $(1.9 \pm 0.3 \%)$, spathulenol $(1.7 \pm 0.1 \%)$, caryophyllene oxide $(1.4 \pm 0.1 \%)$, and $\gamma$-muurolene $(1.2 \pm 0.2 \%)$ were also detected. Sesquiterpene content was found relatively higher in AEO (3.7\%), whereas monoterpene content was meager $(0.4 \%)$. Volatile composition of MREO showed 
abundance of furanoeudesm (41.9\%) and curcerene (23.9\%), considered as marker components of MREO. Besides these, other sesquiterpenoids such as $\beta$-elemene $(4.8 \%), \delta$-elemene $(2.1 \%)$, germacrene $\mathrm{D}(1.2 \%)$, elemol acetate $(1.2 \%)$, and $\beta$-cudesmol (1.1\%) were also identified.

Total ion chromatogram (TIC) of WEO in GC-MS analysis exhibited characteristic peaks corresponding to 29 mono- and sesquiterpenes, contributing $97.7 \%$ of the oil. Oxygenated terpenoids (69.7\%) formed the major share of the composition; borneol acetate $(26.6 \pm 1.7 \%)$ and $\beta$-terpineol $(16.2 \pm 0.9 \%)$ being the most dominant ones. 1,8-Cineol (10.6 $\pm 0.5 \%)$, linalool $(6.4 \pm 0.4 \%)$, sabinene $(5.6 \pm 0.3 \%)$, o-cymene $(5.1 \pm 0.2 \%)$, camphor $(3.8 \pm 0.5 \%)$, and $\alpha$-pinene $(2.7 \pm 0.2 \%)$ were other important terpenes identified in WEO. Sesquiterpenoids
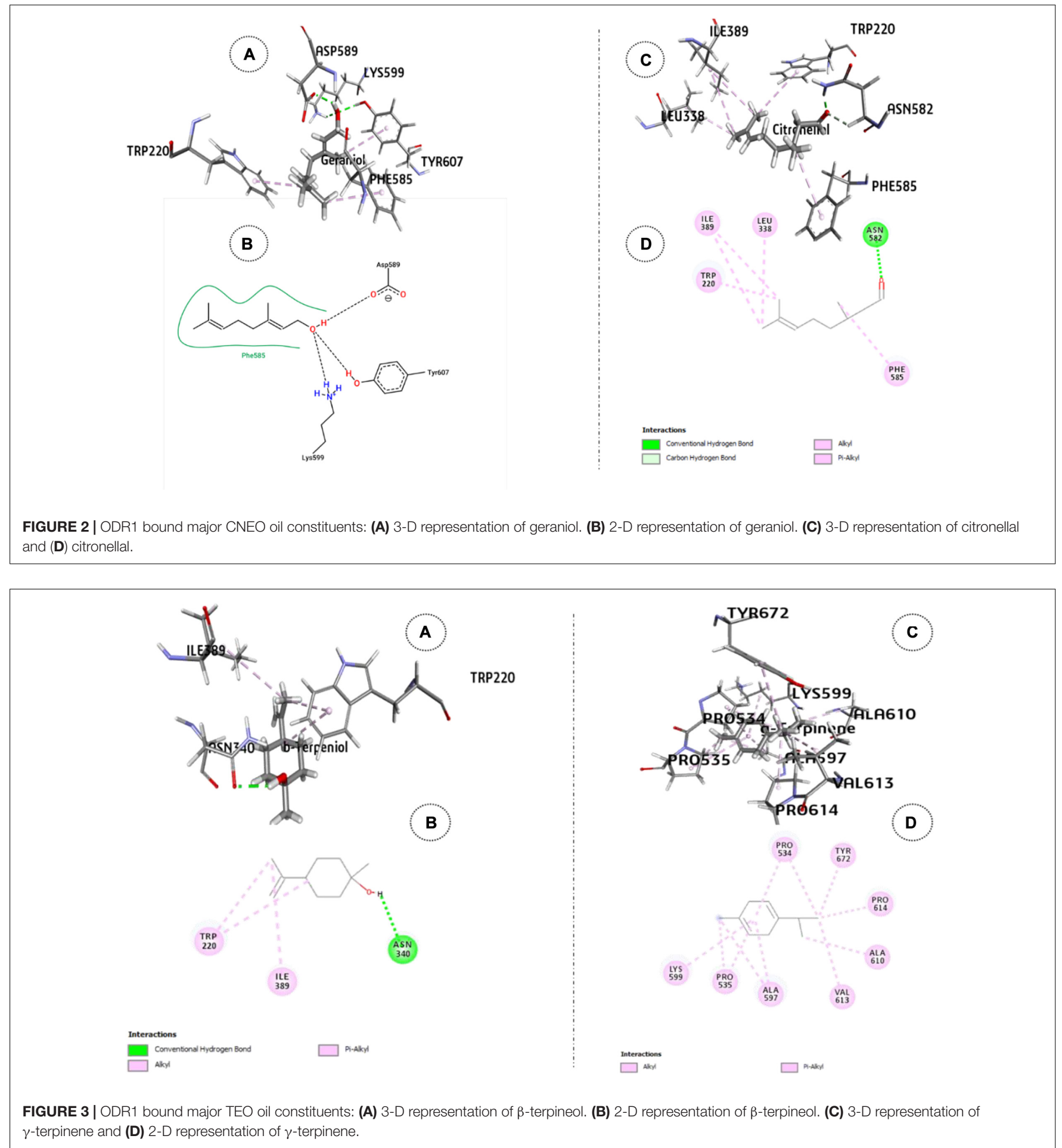
detected in WEO were $\beta$-caryophyllene ( $8.2 \pm 1.0 \%)$, farnesol $(3.0 \pm 0.2 \%)$, and $\delta$-elemene $(2.0 \pm 0.3 \%)$. Volatile composition of PEO showed various peaks in TIC of GC-MS, representing 15 constituents contributing $97.1 \%$ of the oil. Sesquiterpene constituents were highly abundant. Among these, $\alpha$-guaiene $(40.6 \pm 1.3 \%)$ was the major compound followed by $\alpha$-bulnesene (14.6 $\pm 0.9 \%), \alpha$-patchoulene (10.7 $\pm 0.5 \%)$, patchoulol $(6.7 \pm 0.3 \%)$, and $\beta$-patchoulene $(5.8 \pm 0.5 \%)$. A comprehensive profile of the chemical composition of the EOs, number of identified compounds, and their group-wise classification is presented in Table 3, which described the number of compounds identified along with their content based on functional groups.

\section{Nematicidal Activity of Essential Oils}

All the test EOs immobilized more than 50\% of juveniles of M. incognita at different test concentrations. Antinemic activity of the EOs is depicted in Table 4. CNEO exhibited $\mathrm{LC}_{50}$ of $325.41,87.27$, and $43.22 \mu \mathrm{g} \mathrm{ml}^{-1}$ concentration after 24,48 , and 72-h exposure, respectively. However, CEO containing a high amount of citronellal showed moderate activity with an $\mathrm{LC}_{50}$ of $124.50 \mu \mathrm{g} \mathrm{ml}^{-1}$ after $72 \mathrm{~h}$. OEO rich in $l$-limonene was found to exhibit a comparatively higher nematode toxicity with a lethal concentration $\mathrm{LC}_{50}$ of $353.20 \mu \mathrm{g} \mathrm{ml}^{-1}$ within $24 \mathrm{~h}$ of $\mathrm{J}_{2}$ exposure. The nematicidal activity of OEO enhanced with the exposure time, and the highest activity was recorded at an $\mathrm{LC}_{50}$ of 79.35 and $39.37 \mu \mathrm{g} \mathrm{ml}^{-1}$ after 48 and $72 \mathrm{~h}$, respectively (Table 4).

In this study, TEO and AEO were found effective with an $\mathrm{LC}_{50}$ of 76.28 and $85.23 \mu \mathrm{g} \mathrm{ml}^{-1}$ within $72 \mathrm{~h}$, whereas CEO exhibited an $\mathrm{LC}_{50}$ of $124.50 \mu \mathrm{g} \mathrm{ml}^{-1}$. MTEO, however, exerted moderate action with an $\mathrm{LC}_{50}$ of $879.40 \mu \mathrm{g} \mathrm{ml}^{-1}$. The first three Eos, i.e., OEO, CNEO, and TEO, with an $\mathrm{LC}_{50}(72 \mathrm{~h})$ below $50 \mu \mathrm{g} \mathrm{ml}^{-1}$ except TEO, were subjected to molecular docking analysis, to understand their possible interaction with proteins for nematicidal action.

\section{Molecular Docking Study}

Seven receptor proteins (putative target proteins) of $M$. incognita were screened against the biomolecules of OEO, CNEO, and TEO, the three most effective EOs in the present study. Gibb's free energy of binding and other docking parameters of the screened targets are presented in Table 5. Bioactivity of OEO, TEO, and CNEO against $M$. incognita $\mathrm{J}_{2} \mathrm{~s}$ was best explained by the in silico inhibition of the odorant response gene 1 (ODR1). The binding pocket of the ODR 1 allosteric site is composed of 45 amino acid residues. Screening of the compounds present in the three EOs against the ODR1 gave significantly low binding free energy values ranging from -36.9 to $15.3 \mathrm{kcal} \mathrm{mol}^{-1}$, suggestive of formation of stable protein-ligand complexes.

The relative stability of the docked complexes of 49 ligands (major compounds, $>10 \%$ present in the OEO, TEO, and CNEO oils) with the ODR1 was computed in terms of ligand efficiency (Table 5). It can be seen that the lowest binding energy value for the geraniol-ODR1 complex $\left(-36.9 \mathrm{kcal} \mathrm{mol}^{-1}\right)$, as depicted in Table 6 may be attributed to the three conventional $\mathrm{H}$-bonds with relatively shorter bond distances $(\sim 2 \AA)$. Additionally, it appeared that the three hydrophobic interactions of $\pi$-alkyl type led to further stabilization of the geraniol-ODR1 complex (Table 7). Geraniol was bound specifically to the guanylate cyclase catalytic domain of the ODR1 receptor (Figure 2).

The citronellal-ODR1 complex with two $\mathrm{H}$ bonds (one conventional and one $\mathrm{C}-\mathrm{H}$ type) and five hydrophobic bonds (three alkyl and two $\pi$-alkyl types) (Figure 2), exhibited $\Delta \mathrm{G}$ $29.3 \mathrm{kcal} \mathrm{mol}^{-1}$. In this case, the amino acid residues responsible for the ligand binding interactions belonged to both guanylate cyclase and tyrosine/serine/threonine kinase catalytic domains.

The $\beta$-terpineol-ODR1 complex, emerged as the next strongest one with $-32.7 \mathrm{kcal} \mathrm{mol}^{-1}$ binding energy. One conventional $\mathrm{H}$ bond and three hydrophobic bonds (one alkyl type and two $\pi$-alkyl types) attributed to complex formation

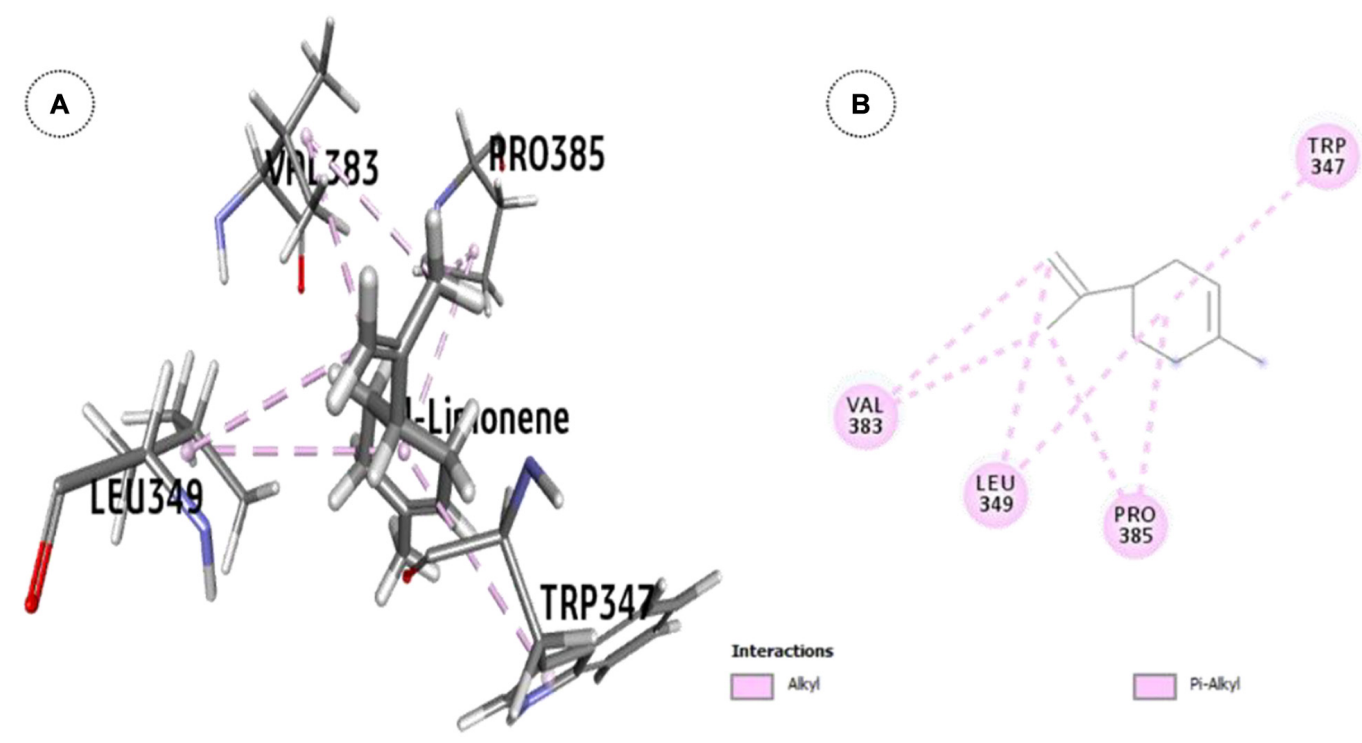

FIGURE 4 | ODR1 bound major OEO oil constituent: (A) 3-D representation of /-limonene and (B) 2-D representation of /-limonene. 
(Figure 3). Here, the specific binding site was tyrosine and serine/threonine kinase catalytic domain.

$l$-Limonene, the major constituent $(93.2 \% \mathrm{w} / \mathrm{w})$ in OEO showed significant inhibition of the ODR1 gene $(\Delta G=$ $\left.25.7 \mathrm{kcal} \mathrm{mol}^{-1}\right)$. The -limonene-ODR1 complex exhibited seven hydrophobic interactions (six alkyl and one $\pi$-alkyl type) in between the protein and the ligand (Figure 4). Apparently, it was bound to the tyrosine/serine/threonine kinase catalytic domain.

The next major EO constituent showing significantly low free energy of binding was $\gamma$-terpinene $\left(\Delta \mathrm{G}=-24 \mathrm{kcal} \mathrm{mol}^{-1}\right.$, $17.5 \%$ in TEO). The $\gamma$-terpinene-ODR1 complex showed 10 hydrophobic interactions (nine alkyl and one $\pi$-alkyl type). The $\gamma$-terpinene molecule bound to the HNOBA and guanylate cyclase catalytic domains.

Based on the observed relative $\Delta G$ values of ligand receptor complexes, $l$-limonene ranked fourth (geraniol-ODR1 complex $>\beta$-terpineol-ODR1 complex $>$ citronellal-ODR1 complex $>l$-limonene-ODR1 complex $>\gamma$-terpinene-ODR1 complex). Inspite of this, the highest observed in vitro nematicidal activity of the OEO oil with $l$-limonene could be possible due to the exceptionally high content of $l$-limonene (93.7\%). The major constituents in other active EOs was up to about $36 \%$ only (Table 6 ).

In order to compare the efficiency of smaller ligands with larger ligands in a non-biased manner, ligand efficiency (LE) calculated for the 49 phytochemical constituents of the three oils varied in the range of $0.8-0.24 \mathrm{kcal} \mathrm{mol}^{-1} \mathrm{HA}^{-1}$. Ninety-one percent of the compounds had an LE above the threshold value of $0.3 \mathrm{kcal} \mathrm{mol}^{-1} \mathrm{HA}^{-1}$, establishing the discovery of natural leads targeting the ODR1 gene in M. incognita. This is the first report on the quantitative binding affinity of the EO constituents toward the ODR1 gene of the root-knot nematode, $M$. incognita, to the best of our information.

\section{DISCUSSION}

In the present study, we performed comprehensive chemoprofiling of EOs in order to understand their possible interactions with the target sites of $M$. incognita. The previously investigated reports on $\mathrm{OEO}$ suggested the most prominent monoterpene, l-limonene, with a range of 32-98\% (Zhang et al., 2019; Matuka et al., 2020). Dejam and Farahmand (2017) described MTEO as primarily composed of monoterpenes such as 1,8-cineol, $\alpha$-pinene, and linalool, which was further confirmed in our study. However, Tunisian MTEO have been reported to be rich in $\alpha$-pinene (Jamoussi et al., 2005). In our study, $\alpha$ pinene has been found to be a major component of MTEO. Bioactive terpenic compositions of CEO make it worthy to study on volatile constituents for diverse biological properties. Contrastingly, the oil contains a high amount of citronellal, citronellol, and isopulegol (Singh et al., 2012; Siddique et al., 2013). An earlier report by Madalosso et al. (2017) and Raymond et al. (2017) described the volatile composition of TEO rich in terpinenes, terpinen-4-ol, and methyl eugenol. Our analysis too revealed that TEO comprised of $\beta$-terpineol and terpinene. Methyl eugenol, however, was not detected. Our findings on AEO predominantly containing $\beta$-asarone have been corroborated by Deepalakshmi et al. (2016). The present study suggested that industrially important MREO, CNEO, WEO, and PEO contained a higher amount of furanoeudesm 1,3 diene, geraniol, myrcene, camphor, and patchoulol, respectively, as reported previously (Buré and Sellier, 2004; Nguyen et al., 2018; Kalaiselvi et al., 2019). Reported variation in chemical profiles of these EOs could be attributed to the plant sources related to locational, seasonal, and climatic factors.

Plant EOs have been described as having great potential in nematode control (Andrés et al., 2012). Oxygenated monoterpenes particularly aldehydes and alcohols have particularly been found effective against $M$. incognita (Echeverrigaray et al., 2010). A similar trend in activity was demonstrated in the case of CNEO comprising an abundance of citronellal and geraniol (Choi et al., 2007). The activity increased both with increasing concentration of EOs and treatment time. Literature also confirmed that EOs containing higher amounts of $l$-limonene usually showed excellent nematicidal potential (Duschatzky et al., 2004). The relative order of nematicidal activity exhibited by the test EOs after a 72-h incubation period, was $\mathrm{OEO}>\mathrm{CNEO}>\mathrm{TEO}>\mathrm{AEO}>\mathrm{CEO}>\mathrm{PEO}>\mathrm{WEO}>$ MTEO > MREO.

\section{CONCLUSION}

The present study employs analytical and molecular modeling tools to relate the nematicidal activity of potential essential oils and the interactions of their chemical constituents with the target site proteins of the organism. Among the nine essential oils screened against $M$. incognita in vitro, the orange (OEO) and citronella (CNEO) oils were identified in the present work as most effective for immobilization and killing of nematodes. In silico analysis suggested a higher binding capacity of geraniol, $\beta$-terpineol, citronellal, $l$-limonene, $\gamma$-terpinene, to the selected target proteins. Molecular docking-based understanding of the bioactivity of aromatic oils is a novel attempt toward logic-driven selection of natural materials and discovery of biopesticidal leads. The present findings will be further confirmed through wet lab molecular studies and utilized in bionematicide product development.

\section{DATA AVAILABILITY STATEMENT}

The original contributions presented in the study are included in the article/supplementary materials, further inquiries can be directed to the corresponding author/s.

\section{AUTHOR CONTRIBUTIONS}

AS, AdK, AD, and AM conceptualized the study. AdK, AM, AnK, and SD validated the study. AdK, AD, AM, LN, RP, MM, JA, NP, and PS conducted the investigation. AS provided the resources. AdK, AM, AD, and SM wrote and prepared the original 
draft. AdK, AM, AD, SS, and RK wrote, reviewed, and edited the manuscript. AS and UR supervised the study. AS acquired the funding. All authors contributed to the article and approved the submitted version.

\section{FUNDING}

This work was supported by the Indian Council of Agricultural Research-Niche Area of Excellence Programme (ICARNAE) (Grant number: Edn. 5(17)/2017-EP\&HS), Ministry of

\section{REFERENCES}

Abagyan, R., Totrov, M., and Kuznetsov, D. (1994). ICM-a new method for protein modeling and design: applications to docking and structure prediction from the distorted native conformation. J. Comput. Chem. 15, 488-506. doi: 10.1002/jcc.540150503

Aissani, N., Balti, R., and Sebai, H. (2018). Potent nematicidal activity of phenolic derivatives on Meloidogyne incognita. J. Helminthol. 92, 668-673. doi: 10.1017/ s0022149x17000918

Aissani, N., Urgeghe, P. P., Oplos, C., Saba, M., Tocco, G., Petretto, G. L., et al. (2015). Nematicidal activity of the volatilome of Eruca sativa on Meloidogyne incognita. J. Agricult. Food Chem. 63, 6120-6125.

Amora, D. X., de Podesta, G. S., Nasu, E. D. G. C., de Figueiredo, L. D., Ferreira, F. C., Lopes, E. A., et al. (2017). Effect of essential oils on the root-knot nematode management. Agri-Environ. Sci. 3, 15-23.

Andrés, M. F., González-Coloma, A., Sanz, J., Burillo, J., and Sainz, P. (2012). Nematicidal activity of essential oils: a review. Phytochem. Rev. 11, 371-390. doi: 10.1007/s11101-012-9263-3

Ardakani, A. S., Hosyninejad, S. A., and Pourshirzad, A. (2013). Killing effects of Myrtus communis L. essential oil on Meloidogyne incognita. Int. J. Agric. Crop Sci. 5, 806-810.

Atolani, O., and Fabiyi, O. A. (2020). "Plant parasitic nematodes management through natural products: current progress and challenges," in Management of Phytonematodes: Recent Advances and Future Challenges, eds R. Ansari, R. Rizvi, and I. Mahmood (Singapore: Springer), 297-315. doi: 10.1007/978-98115-4087-5_13

Avato, P., Laquale, S., Argentieri, M. P., Lamiri, A., Radicci, V., and D’Addabbo, T. (2017). Nematicidal activity of essential oils from aromatic plants of Morocco. J. Pest Sci. 90, 711-722. doi: 10.1007/s10340-016-0805-0

Bakkali, F., Averbeck, S., Averbeck, D., and Idaomar, M. (2008). Biological effects of essential oils - a review. Food Chem. Toxicol. 46, 446-475.

Buré, C. M., and Sellier, N. M. (2004). Analysis of the essential oil of Indonesian patchouli (Pogostemon cablin Benth.) using C/MS (EI/CI). J. Essent. Oil Rese. 16, 17-19. doi: 10.1080/10412905.2004.9698638

Bursulaya, B. D., Totrov, M., Abagyan, R., and Brooks, C. L. (2003). Comparative study of several algorithms for flexible ligand docking. J. Comput. Aided Mol. Des. 17, 755-763. doi: 10.1023/b:jcam.0000017496.76572.6f

Caboni, P., Saba, M., Tocco, G., Casu, L., Murgia, A., Maxia, A., et al. (2013). Nematicidal activity of mint aqueous extracts against the root-knot nematode Meloidogyne incognita. J. Agric. Food Chem. 61, 9784-9788. doi: 10.1021/ jf403684h

Cantrell, C. L., Dayan, F. E., and Duke, S. O. (2012). Natural products as sources for new pesticides. J. Nat. Prod. 75, 1231-1242. doi: 10.1021/np300024u

Choi, I., Kim, J., Shin, S., and Park, I. (2007). Nematicidal activity of monoterpenoids against the pine wood nematode (Bursaphelenchus xylophilus). Russian J. Nematol. 15, 35-40.

Cobb, N. A. (1918). Estimating the Nema Population of Soil, with Special Reference to the Sugar-beet and Root-gall Nemas, Heterodera schachtii Schmidt and Heterodera radicicola (Greef) Müller: and with a Description of Tylencholaimus Aequalis n. sp. Washington, D.C: US Government Printing Office.

Collange, B., Navarrete, M., Peyre, G., Mateille, T., and Tchamitchian, M. (2011). Root-knot nematode (Meloidogyne) management in vegetable crop production:
Agriculture, New Delhi, India. Though there was no funds from the funding agency for article processing for open access publication fees, authors will pay themselves. Thus, all the authors requested if the charges can be discounted at the maximum.

\section{ACKNOWLEDGMENTS}

The authors wish to thank the Director, Indian Council of Agricultural Research-Indian Agricultural Research Institute (ICAR-IARI), New Delhi, India.

the challenge of an agronomic system analysis. Crop Protec. 30, 1251-1262. doi: 10.1016/j.cropro.2011.04.016

de Freitas Silva, M., Campos, V. P., Barros, A. F., da Silva, J. C. P., Pedroso, M. P., de Jesus Silva, F., et al. (2020). Medicinal plant volatiles applied against the root-knot nematode Meloidogyne incognita. Crop Prot. 130:105057. doi: 10.1016/j.cropro.2019.105057

Deepalakshmi, P. D., Odgerel, K., Thirugnanasambantham, P., Yungeree, O., Khorolragchaa, A., and Senthil, K. (2016). Metabolite profiling of in vitro cultured and field grown rhizomes of Acorus calamus from Mongolia using GC-MS. Chromatographia 79, 1359-1371. doi: 10.1007/s10337-016-3152-7

Dejam, M., and Farahmand, Y. (2017). Essential oil content and composition of myrtle (Myrtus communis L.) leaves from South of Iran. J. Essent. Oil Bearing Plants 20, 869-872. doi: 10.1080/0972060x.2014.981599

Dunbrack, R. L. Jr. (2006). Sequence comparison and protein structure prediction. Curr. Opin. Struct. Biol. 16, 374-384. doi: 10.1016/j.sbi.2006.05.006

Duschatzky, C. B., Martinez, A. N., Almeida, N. V., and Bonivardo, S. L. (2004). Nematicidal activity of the essential oils of several Argentina plants against the root-knot nematode. J. Essent. Oil Res. 16, 626-628. doi: 10.1080/10412905. 2004.9698812

Echeverrigaray, S., Zacaria, J., and Beltrão, R. (2010). Nematicidal activity of monoterpenoids against the root-knot nematode Meloidogyne incognita. Phytopathol 100, 199-203. doi: 10.1094/phyto-100-2-0199

Hopkins, A. L., Keserü, G. M., Leeson, P. D., Rees, D. C., and Reynolds, C. H. (2014). The role of ligand efficiency metrics in drug discovery. Nat. Rev. Drug Discov. 13, 105-121. doi: 10.1038/nrd4163

Jamoussi, B., Romdhane, M., Abderraba, A., Hassine, B. B., and Gadri, A. E. (2005). Effect of harvest time on the yield and composition of Tunisian myrtle oils. Flavour Frag. J. 20, 274-277. doi: 10.1002/ffj.1453

Jones, J. G., Kleczewski, N. M., Desaeger, J., Meyer, S. L., and Johnson, G. C. (2017). Evaluation of nematicides for southern root-knot nematode management in lima bean. Crop Prot. 96, 151-157. doi: 10.1016/j.cropro.2017.02.015

Julio, L. F., González-Coloma, A., Burillo, J., Diaz, C. E., and Andrés, M. F. (2017). Nematicidal activity of the hydrolate byproduct from the semi industrial vapor pressure extraction of domesticated Artemisia absinthium against Meloidogyne javanica. Crop Prot. 94, 33-37. doi: 10.1016/j.cropro.2016.12.002

Kalaiselvi, D., Mohankumar, A., Shanmugam, G., Thiruppathi, G., Nivitha, S., and Sundararaj, P. (2019). Altitude-related changes in the phytochemical profile of essential oils extracted from Artemisia nilagirica and their nematicidal activity against Meloidogyne incognita. Ind. Crop Prod. 139:111472. doi: 10.1016/j. indcrop.2019.111472

Kalemba, D., and Kunicka, A. (2003). Antibacterial and antifungal properties of essential oils. Curr. Med. Chem. 10, 813-829. doi: 10.2174/0929867033457719

Kong, J. O., Lee, S. M., Moon, Y. S., Lee, S. G., and Ahn, Y. J. (2006). Nematicidal activity of plant essential oils against Bursaphelenchus xylophilus (Nematoda: Aphelenchoididae). J. Asia-Pac. Entomol. 9, 173-178. doi: 10.1016/s12268615(08)60289-7

Kundu, A., Saha, S., Walia, S., and Dutta, T. K. (2016). Antinemic potentiality of chemical constituents of Eupatorium adenophorum Spreng leaves against Meloidogyne incognita. Nat. Acad. Sci. Lett. 39, 145-149. doi: 10.1007/s40009016-0439-z

Lee, Y. S., Kim, J., Lee, S. G., Oh, E., Shin, S. C., and Park, I. K. (2009). Effects of plant essential oils and components from Oriental sweetgum (Liquidambar 
orientalis) on growth and morphogenesis of three phytopathogenic fungi. Pestic. Biochem. Physiol. 93, 138-143. doi: 10.1016/j.pestbp.2009.02.002

Madalosso, R. G., Verdi, C. M., Souza, M. E., Cunha, J. A., Santos, P. S., Balardin, R. S., et al. (2017). In vitro and in vivo effects of Melaleuca alternifolia nanoparticles in Meloidogyne sp. J. Asia-Pac. Entomol. 20, 1344-1349. doi: 10.1016/j.aspen.2017.10.002

Matuka, T., Oyedeji, O., Gondwe, M., and Oyedeji, A. (2020). Chemical composition and in vivo anti-inflammatory activity of essential oils from Citrus sinensis (L.) osbeck growing in South Africa. J. Essent. Oil Bear. Plants 23, 638-647. doi: 10.1080/0972060x.2020.1819885

Neves, M. A., Totrov, M., and Abagyan, R. (2012). Docking and scoring with ICM: the benchmarking results and strategies for improvement. J. Comput. Aided Mol. Des. 26, 675-686. doi: 10.1007/s10822-012-9547-0

Nguyen, H. T., Radácsi, P., Gosztola, B., Rajhárt, P., and Németh, ÉZ. (2018). Accumulation and composition of essential oil due to plant development and organs in wormwood (Artemisia absinthium L.). Ind. Crop Prod. 123, 232-237. doi: $10.1016 /$ j.indcrop.2018.06.076

Oka, Y. (2001). Nematicidal activity of essential oil components against the rootknot nematode Meloidogyne javanica. Nematol. 3, 159-164. doi: 10.1163/ 156854101750236286

Pandey, R., Kalra, A., Tandon, S., Mehrotra, N., Singh, H. N., and Kumar, S. (2000). Essential oils as potent source of nematicidal compounds. J. Phytopathol. 148, 501-502. doi: 10.1046/j.1439-0434.2000.00493.x

Pedroso, L. A., Campos, V. P., Pedroso, M. P., Barros, A. F., Freire, E. S., and Resende, F. M. (2019). Volatile organic compounds produced by castor bean cake incorporated into the soil exhibit toxic activity against Meloidogyne incognita. Pest Manag. Sci. 75, 476-483. doi: 10.1002/ps.5142

Perrett, S., and Whitfield, P. J. (1995). Anthelmintic and pesticidal activity of Acorus gramineus (Araceae) is associated with phenylpropanoid asarones. Phytother. Res. 9, 405-409. doi: 10.1002/ptr.2650090604

Raymond, C. A., Davies, N. W., and Larkman, T. (2017). GC-MS method validation and levels of methyl eugenol in a diverse range of tea tree (Melaleuca alternifolia) oils. Anal. Bioanal. Chem. 409, 1779-1787. doi: 10.1007/s00216016-0134-4

Sidhu, H. S., Kumar, V., and Madhu, M. R. (2017). Eco-Friendly management of root-knot nematode, Meloidogyne javanica in okra (Abelmoschus esculentus) crop. Int. J. Pure Appl. Biosci. 5, 569-574. doi: 10.18782/2320-7051.2507
Siddique, Y. H., Mujtaba, S. F., Jyoti, S., and Naz, F. (2013). GC-MS analysis of Eucalyptus citriodora leaf extract and its role on the dietary supplementation in transgenic Drosophila model of Parkinson's disease. Food Chem. Toxicol. 55, 29-35. doi: 10.1016/j.fct.2012.12.028

Silva, J. C. P., Campos, V. P., Barros, A. F., Pedroso, M. P., Terra, W. C., Lopez, L. E., et al. (2018). Plant volatiles reduce the viability of the root-knot nematode Meloidogyne incognita either directly or when retained in water. Plant Dis. 102, 2170-2179. doi: 10.1094/pdis-01-18-0143-re

Singh, H. P., Kaur, S., Negi, K., Kumari, S., Saini, V., Batish, D. R., et al. (2012). Assessment of in vitro antioxidant activity of essential oil of Eucalyptus citriodora (lemon-scented Eucalypt; Myrtaceae) and its major constituents. LWT Food Sci. Technol. 48, 237-241. doi: 10.1016/j.lwt.2012.03.019

Sinha, A., Maheshwari, R. C., Dureja, P., and Mojumder, V. (2006). Nematicidal activity of essential oils and their components against the root-knot nematode Meloidogyne incognita. Ind. J. Nematol. 36, 109-114.

Tetali, S. D. (2019). Terpenes and isoprenoids: a wealth of compounds for global use. Planta 249, 1-8. doi: 10.1007/s00425-018-3056-x

Westphal, A. (2011). Sustainable approaches to the management of plant-parasitic nematodes and disease complexes. J. Nematol. 43, 122-125.

Zhang, L. L., Yang, Z. Y., Fan, G., Ren, J. N., Yin, K. J., and Pan, S. Y. (2019). Antidepressant-like effect of Citrus sinensis (L.) Osbeck essential oil and its main component limonene on mice. J. Agric. Food Chem. 67, 13817-13828. doi: $10.1021 /$ acs.jafc.9b00650

Conflict of Interest: The authors declare that the research was conducted in the absence of any commercial or financial relationships that could be construed as a potential conflict of interest.

Copyright ( 2021 Kundu, Dutta, Mandal, Negi, Malik, Puramchatwad, Antil, Singh, Rao, Saha, Kumar, Patanjali, Manna, Kumar, Dash and Singh. This is an open-access article distributed under the terms of the Creative Commons Attribution License (CC BY). The use, distribution or reproduction in other forums is permitted, provided the original author(s) and the copyright owner(s) are credited and that the original publication in this journal is cited, in accordance with accepted academic practice. No use, distribution or reproduction is permitted which does not comply with these terms. 